\title{
Relationship between root apices of maxillary posterior teeth and the maxillary sinus floor in patients from the Varna region
}

\section{Tihomir Georgiev ${ }^{1}$, Stefan Peev $^{2}$, Hristina Arnautska ${ }^{3}$.}

1. Department of Oral and Maxillofacial Surgery, Faculty of Dental Medicine, Medical University Varna;

2. Department of Periodontology, Faculty of Dental Medicine, Medical University Varna;

3. Department of Prosthetic Dental Medicine and Orthodontics, Faculty of Dental Medicine, Medical University Varna;

\footnotetext{
Abstract

Pneumatization of the maxillary sinus leads to posterior root displacement into the sinus cavity associated with potential complications following extraction of these teeth.

Objectives. The aim of the present study is to assess the relationship between the apices of maxillary posterior teeth and the floor of the maxillary sinus.

Materials and methods. The study involved a retrospective randomized analysis of 245 scans of the maxilla, 465 scans of sinuses and 960 scans of teeth and their relationship to the maxillary sinus. The distance between root apices and the maxillary sinus floor was measured and the measurements featured canines, premolars and molars.

Results. 746 teeth, out of the 960 teeth examined, were in dangerous proximity to the maxillary sinus, 156 of which penetrated the sinus cavity at different depths.
} 
Conclusions. In patients from the Varna region maxillary second molars appeared to be the most common teeth to project into the sinus and when extracting these teeth dental clinicians must be particularly cautious for possible complications related to this problem.

Keywords: CBCT, apex of the root, sinus floor, molars, premolars, canines

\section{Introduction}

The extraction of posterior maxillary teeth has always proved challenging to dental clinicians due to the proximity of the maxillary sinus and the possibility of encountering a number of complications, either of intraoperative or postoperative nature (1). Anatomically, the maxillary sinus is universally classified into a sclerotic, pneumatic and mixed types (2). Dental clinicians are particularly interested in patients with a highly pneumatized maxillary sinus $(3,4)$. Most often the root apices of the molars of such patients are close to the maxillary sinus and a fairly large percentage of those teeth penetrate the sinus to varying degrees (5). There is evidence that premolars and even canines can be in contact with the maxillary sinus. Scans using Cone beam computed tomography (CBCT) greatly assist in the diagnosis of this relationship $(6,7,8)$.

\section{Material and Methods}

The present study is based on the analysis of a random selection of 245 scans of the maxilla, 465 scans of the maxillary sinus and 960 teeth scans of patients who called at the University Medical and Dental Centre (UMDC) of the Faculty of Dental Medicine at the Medical University Varna. The patients reported at the Centre with various complaints in the upper jaw or in relation to a scheduled implant placement in the distal region. The scans were recorded over a period from 2014 to 2015.

Patients had signed Informed Consent Forms, agreeing on subsequent use of their scans for the purposes of research. The images were acquired with a Cone beam computed tomography (CBCT) scanner (Planmeca ProMax 3D Max), integrated with a computer hard drive and backup peripheral devices, software for reconstructing images (Planmeca Romexis) and a patients registration program. Scanning parameters were: scanning time 9-40 sec., image reconstruction time 2-55 sec. CBCT images were stored and proofread by Planmeca Romexis software, compatible with Windows XP, Windows 7 and Windows 8.

The present study is retrospective and the following measurements were subsequently performed on the patients' scans: the relationship between the root apices of maxillary posterior teeth (from the canine to the wisdom tooth) and the floor of the maxillary sinus (Figure 1). With teeth having two or three roots, the relationships between each root apex and the floor of the maxillary sinus is evaluated separately, and the value of the apex penetrated furthest is recorded for the purposes of this study. The research excluded teeth that were erupting at the time or were ectopically erupted or had not taken their position in the dental row. The study did not include teeth positioned at more than $4 \mathrm{~mm}$ further from the maxillary sinus. These comprised 35 teeth. Teeth were divided into 4 groups, respectively: Group I comprised teeth positioned at a distance of 2-4 $\mathrm{mm}$ from the maxillary sinus; Group II included teeth at 0-2 $\mathrm{mm}$ from the maxillary sinus (with no visible penetration); Group III consisted of teeth with penetrating apices $0-2 \mathrm{~mm}$ into the sinus; Group IV comprised teeth with apices penetrating 2-4 mm into the sinus. The FDI World Dental Federation's dental notation has been adopted. Measurements were taken by two lecturers from UMDC, 
working independently of each other. Whenever there was disagreement on the parameters under study, then a dental radiologist was consulted. Statistical validity is considered to be $p<0.05$.

Figure 1.

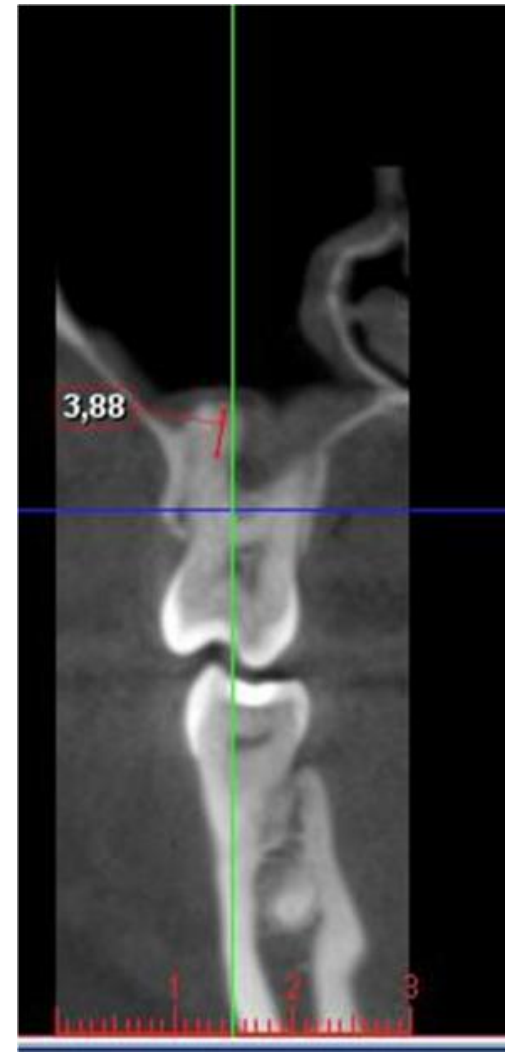

\section{Results}

960 teeth were evaluated, 35 of which were excluded from the study because of their distance from the maxillary sinus being more than $4 \mathrm{~mm}$, as well as other 16 teeth which were ectopically erupted or had not taken their place in the dental row. The youngest patient was 16 years old, the eldest was 82 years old. The target group comprised 909 teeth divided into groups: 10 canines; 53 first premolars; 218 second premolars; 258first molars; 289 second molars; 81 wisdom teeth. All of these teeth were divided into 4 groups, respectively: Group I comprised teeth positioned at a distance of 2-4 $\mathrm{mm}$ from the maxillary sinus; Group II included teeth at 0-2 $\mathrm{mm}$ from the maxillary sinus (with no visible penetration); Group III consisted of teeth with penetrating apices $0-2 \mathrm{~mm}$ into the sinus; Group IV comprised teeth with apices penetrating 2-4 mm into the sinus.

The distribution in groups is as follows:

Group I: 163 teeth in total ( 2 canines, 31 first premolars, 60 second premolars, 30 first molars, 27 second molars, 13 wisdom teeth) See Table 1.

Group II: 590 teeth in total ( 8 canines, 22 first premolars, 143 second premolars, 173 first molars, 192 second molars, 52 wisdom teeth) See Table 2. 
Group III: 141 teeth in total (14 second premolars, 50 first molars, 61 second molars, 16 wisdom teeth) See Table 3.

Group IV: 15 teeth in total (1 second premolar, 5 first molars, 9 second molars) See Table 4.

Figure 2

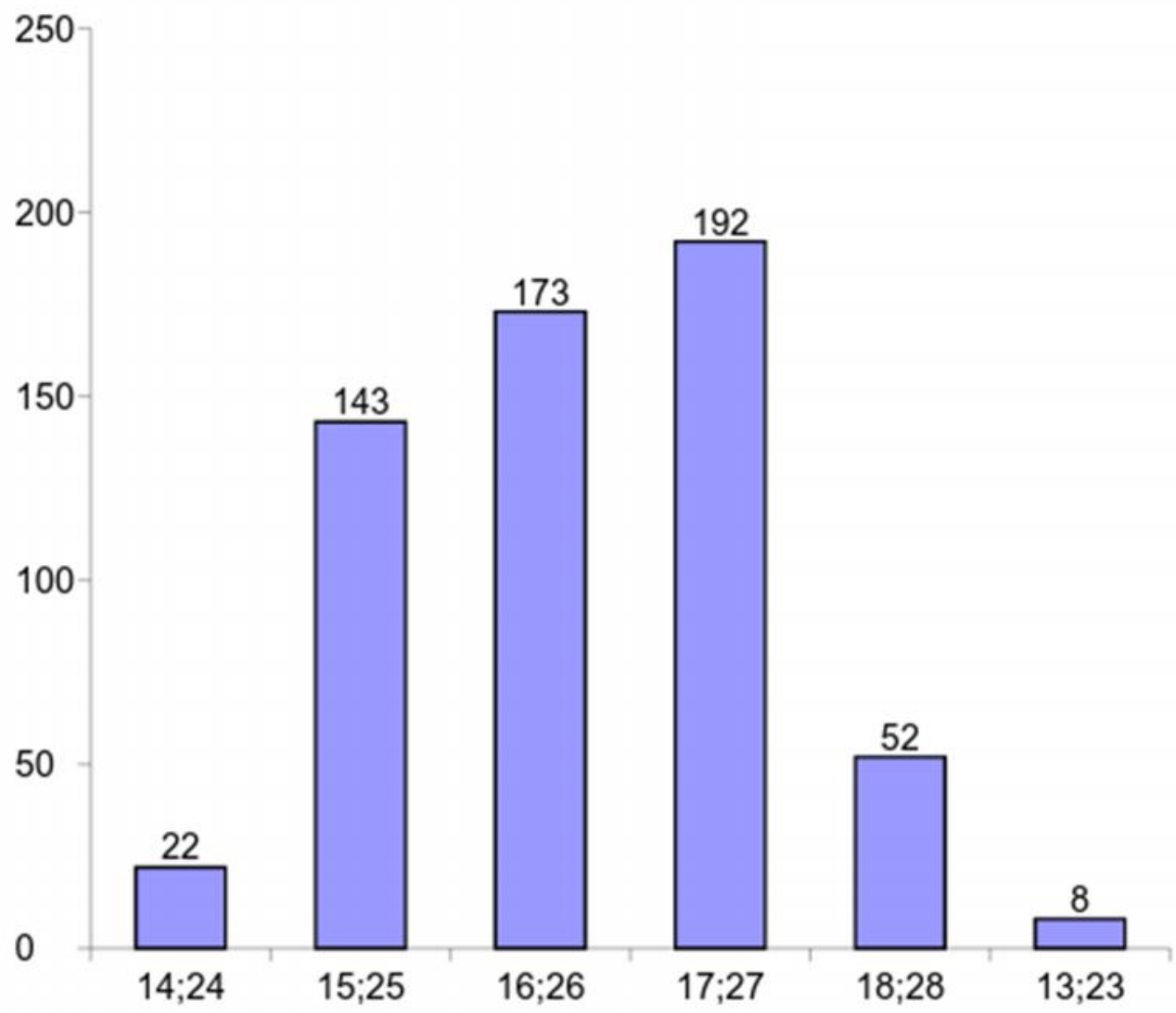

Figure 2 shows the relationship between the different groups of teeth (first premolars - 14,24 ; second premolars - 15, 25; first molars -16, 26 ; second molars - 17,27; wisdom teeth - 18, 28; canines - 13, 23) and the number of teeth at $2-4 \mathrm{~mm}$ from the maxillary sinus floor. 
Figure 3

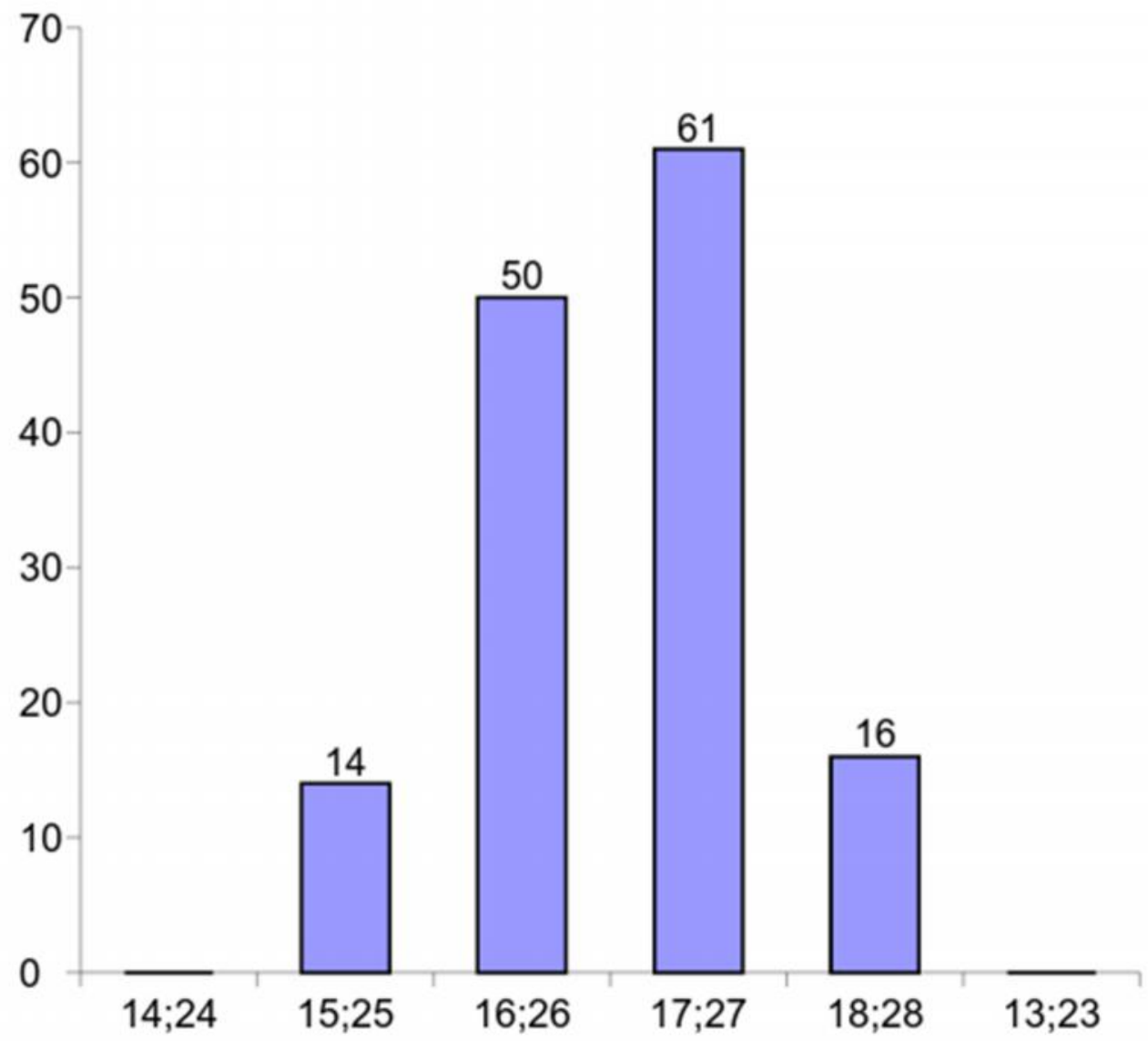

Figure 3 shows the relationship between the different groups of teeth (first premolars - 14, 24; second premolars -15, 25; first molars - 16, 26; second molars - 17, 27; wisdom teeth - 18, 28; canines - 13, 23) and the number of teeth at $0-2 \mathrm{~mm}$ from the maxillary sinus floor. 
Figure 4

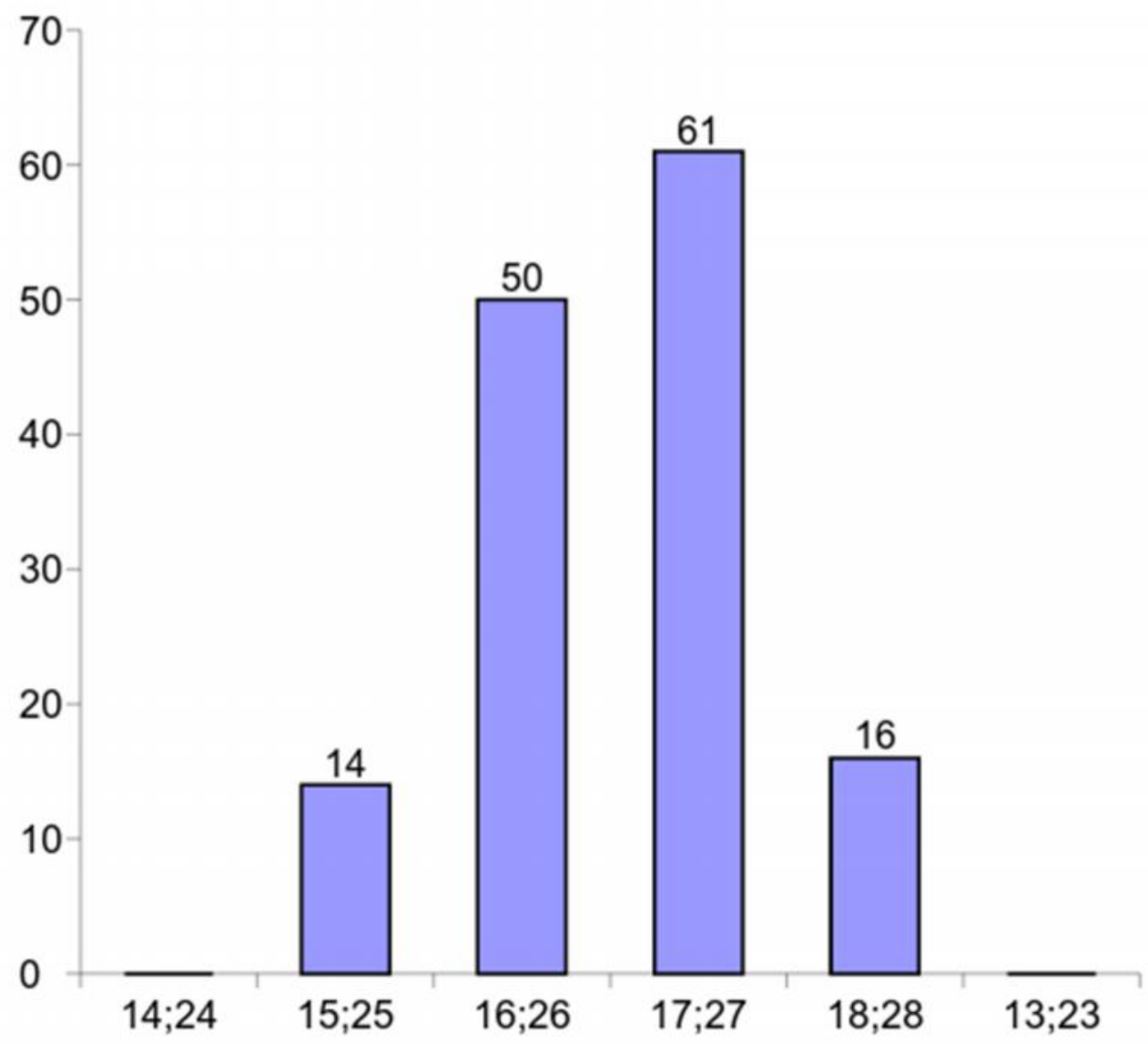

Figure 4 shows the relationship between the different groups of teeth (first premolars - 14, 24; second premolars - 15, 25; first molars - 16, 26; second molars - 17, 27; wisdom teeth - 18, 28; canines - 13, 23) and the number of teeth penetrating $0-2 \mathrm{~mm}$ into the maxillary sinus. 
Figure 5

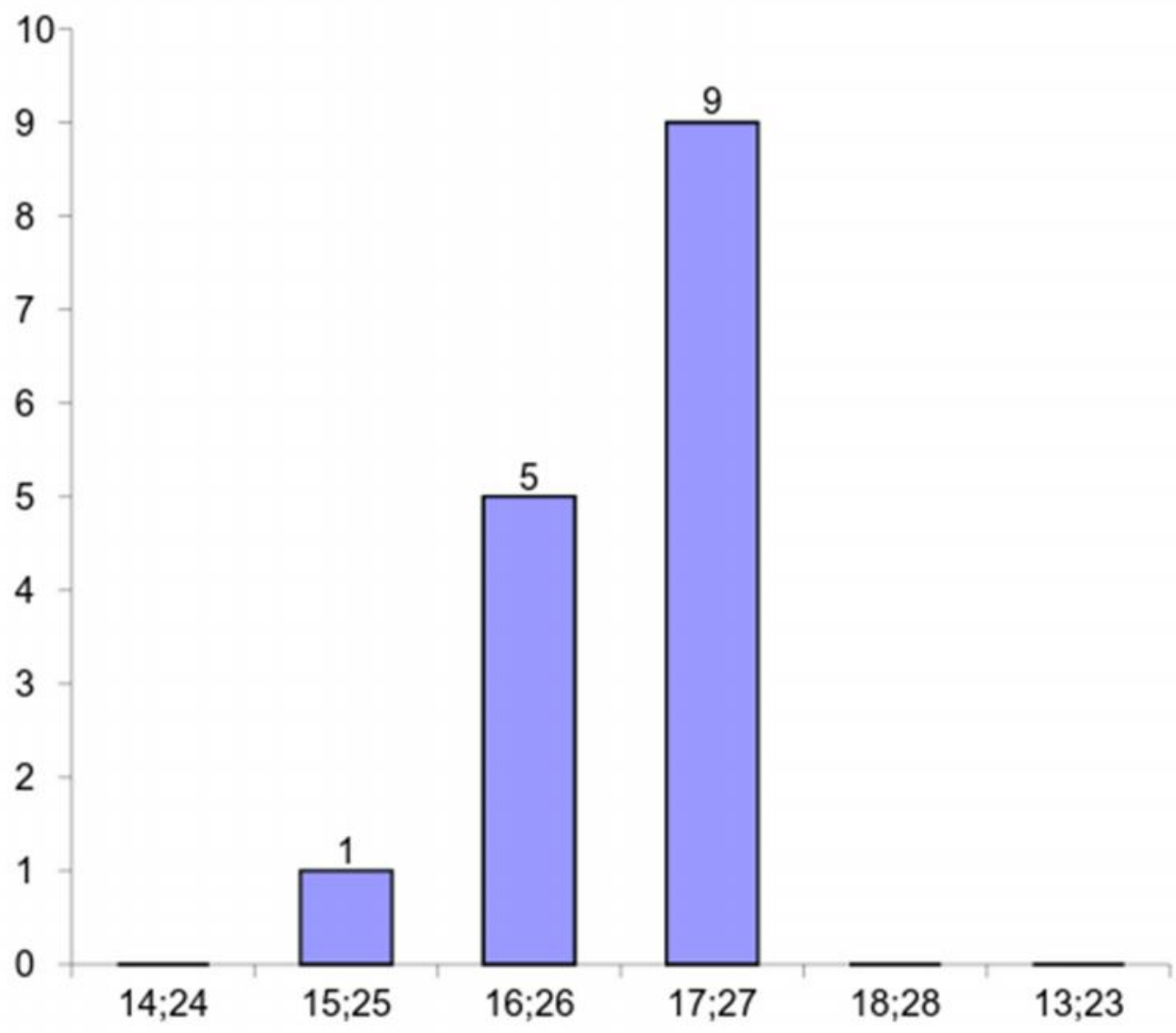

Figure 5 shows the relationship between the different groups of teeth (first premolars - 14, 24; second premolars - 15, 25; first molars - 16, 26; second molars - 17, 27; wisdom teeth - 18, 28; canines - 13, 23) and the number of teeth penetrating $2-4 \mathrm{~mm}$ into the maxillary sinus.

\section{Discussion}

Knowledge of the anatomical relationship betweenthe maxillary sinus floor and the root apices of the maxillary posterior teeth is essential to dental clinicians before planning extraction of these teeth $(9,10,11)$. Complications are associated with creating communication between the maxillary sinus and the oral cavity, possible inflammatory processes which may affect the sinus, such as sinusitis, acute or chronic $(12,13,14,15)$. The relationship between the root apices of the teeth and the inferior sinus allows the dental clinician to assess their own abilities as well as to prepare for potential complications and how to deal with them(16). The purpose of our research was namely to acquire the necessary knowledge as to when, where, and with the extraction of which teeth groups complications could be encountered and how to prepare for their correction.

The study examined the relationship between the apices of mandibular posterior teeth and the sinus floor, including canines which have not been investigated sufficiently using CBCT images. 
In the Group of canine teeth it was observed that 10 teeth werein proximity to the maxillary sinus, 8 of which were at a distance of $0-2 \mathrm{~mm}$ from the sinus floor. This could indicate difficulties with the extraction of those canines and there might be a possibility of complications, such as sinus membrane perforation. 53 first molars were studied, 22 of which appeared dangerously close $(0-2 \mathrm{~mm})$ to the maxillary sinus cavity.

The relationship between the apices of the second premolar roots and the sinus floor posed a greater risk of complications linked to their extraction.

Out of 218 teeth under examination, 143 were dangerously near $(0-2 \mathrm{~mm})$ :the root apices of 14 teeth projected upto 2 mminto the maxillary sinus, whereas 1 tooth even had a 4-mm penetration. In case of penetration, prior to extraction, the dental clinician ought to prepare fo rlocal plastic surgery measuers for closure of accidental communication as a result of the tooth extraction.

First molars (a total of 258 teeth under study) showed a greater risk of accidental perforation of the sinus in case of their extraction: 173 teeth appeared to be in dangerous proximity, 50 teeth projected upto $2 \mathrm{~mm}$ into the sinus and 5 teeth penetrated upto $4 \mathrm{~mm}$ into thesinus.

Second molars proved to have the greatest number close to the sinus or in contact with it:out of a total of 289 teeth, 192 were dangerously close; 61 penetrated upto 2 mmand 9 teeth - upto $4 \mathrm{~mm}$.

Relatively few wisdom teeth were explored in this study due to their often ectopic eruption, their impaction or late eruption: 81 wisdom teeth in total were assessed, 52 of which were close to the maxillary sinus, while 16 penetrated the sinus upto $2 \mathrm{~mm}$.

Out of a total of 960 teeth analyzed, 590 teeth appeared to be in dangerous proximity to the sinus, 141 teeth penetrated the sinus upto $2 \mathrm{~mm}$ and 15 teeth - upto $4 \mathrm{~mm}$. To a dental clinician these findings imply that more than $77.7 \%$ of maxillary posterior teeth could pose problems with their extraction; with more than $14.7 \%$ of the teeth clinicians ought to be $100 \%$ ready to perform local plastic surgery procedures for closure of communication that would be created during the extraction of the teeth penetrating the maxillary sinus. The present research established that the tooth most likely to have root apices penetrating the maxillary sinus is the second molar. This can be explained by the fact that the first molar erupts much earlier in the oral cavity, it is longer subjected to caries processes and consequent complications, inflammatory reactions of the periodontium and as a result it gets extracted earlier. Evidence in support of the above statement is also the number of those teeth investigated: 258 first molars and 289 second molars. This points to the need of a more thorough study to be performed where a larger amount of patients will have to be analyzed.

\section{Conclusion}

Cone beam computed tomography (CBCT) scans prove essential in the proper diagnosis of the relationship between the root apices of maxillary posterior teeth and the maxillary sinus floor. When communication is suspected, a scan of the relevant region is imperative. Teeth that have penetrated the maxillary sinus ought to be extracted by specialists in Oral surgery so that prompt local plastic surgery procedures could be performed for closure of the communication between the oral cavity and the maxillary sinus. 


\section{References}

1. Teng $M$, Cheng Q, Liao J, Zhang X, Mo A, Liang X. Sinus Width Analysis and New Classification with Clinical Implications for Augmentation. Clin Implant Dent Relat Res. 2014 Jul 17. doi: $10.1111 /$ cid. 12247.

2. Shahbazian M, Vandewoude C, Wyatt J, Jacobs R. Comparative assessment of periapical radiography and CBCT imaging for radiodiagnostics in the posterior maxilla. Odontology. 2015 Jan;103(1):97-104. doi: 10.1007/s10266-013-0144-z. Epub 2013 Dec 28.

3. Shiki K, Tanaka T, Kito S, Wakasugi-Sato N, Matsumoto-Takeda S, Oda M, Nishimura S, Morimoto $\mathrm{Y}$. The significance of cone beam computed tomography for the visualization of anatomical variations and lesions in the maxillary sinus for patients hoping to have dental implantsupported maxillary restorations in a private dental office in Japan. Head Face Med. 2014 May 28;10:20. doi: 10.1186/1746-160X-10-20.

4. Ok E, Güngör E, Colak M, Altunsoy M, Nur BG, Ağlarci OS. Evaluation of the relationship between the maxillary posterior teeth and the sinus floor using cone-beam computed tomography. Surg Radiol Anat. 2014 Nov;36(9):907-914. doi: 10.1007/s00276-014-1317-3.

5. von Arx T, Fodich I, Bornstein MM Proximity of premolar roots to maxillary sinus: a radiographic survey using cone-beam computed tomography. J Endod. 2014 Oct;40(10):1541-1548. doi: 10.1016/j.joen.2014.06.022.

6. Inara Carneiro Costa Rege, Thiago Oliveira Sousa, Cláudio Rodrigues Leles, Elismauro Francisco Mendonça Occurrence of maxillary sinus abnormalities detected by cone beam CT in asymptomatic patients Rege et al. BMC Oral Health 2012, 12:30 http://www.biomedcentral.com/1472-6831/12/30

7. Zheng X, Teng M, Zhou F, Ye J, Li G, Mo A Influence of Maxillary Sinus Width on Transcrestal Sinus Augmentation Outcomes: Radiographic Evaluation Based on Cone Beam CT. Clin Implant Dent Relat Res. 2015 Feb 26. doi: 10.1111/cid.12298.

8. Parks ET. Cone beam computed tomography for the nasal cavity and paranasal sinuses. Dent Clin North Am. 2014 Jul;58(3):627-651. doi: 10.1016/j.cden.2014.04.003.

9. Shanbhag S, Shanbhag V, Stavropoulos A. Volume changes of maxillary sinus augmentations over time: a systematic review. Int J Oral Maxillofac Implants. 2014 Jul-Aug;29(4):881-892. doi: 10.11607/jomi.3472.

10. Kretzschmar DP, Kretzschmar JL. Rhinosinusitis: review from a dental perspective. Oral Surg Oral Med Oral Pathol Oral Radiol Endod. 2003 Aug;96(2):128-135.

11. Yun-Hoa Jung, Bong-Hae Cho Assessment of the relationship between the maxillary molars and adjacent structures using cone beam computed tomography Imaging Science in Dentistry 2012; 42 : 219-224, http://dx.doi.org/10.5624/isd.2012.42.4.219 
12. Kilic C, Kamburoglu K, Yuksel SP, Ozen T. An Assessment of the Relationship between the Maxillary Sinus Floor and the Maxillary Posterior Teeth Root Tips Using Dental Cone-beam Computerized Tomography.Eur J Dent. 2010 Oct;4(4):462-467.

13. Lanzer M, Pejicic R, Kruse AL, Schneider T, Grätz KW, Lübbers HT. Anatomic (positional) variation of maxillary wisdom teeth with special regard to the maxillary sinus. Swiss Dent J. 2015;125(5):555-571.

14. Vogiatzi T, Kloukos D, Scarfe WC, Bornstein MM. Incidence of anatomical variations and disease of the maxillary sinuses as identified by cone beam computed tomography: a systematic review. Int J Oral Maxillofac Implants. 2014 Nov-Dec;29(6):1301-1314. doi: 10.11607/jomi.3644.

15. Shokri A, Lari S, Yousef F, Hashemi L. Assessment of the Relationship between the Maxillary Sinus Floor and Maxillary Posterior Teeth Roots using Cone Beam Computed Tomography. J Contemp Dent Pract. 2014 Sep 1;15(5):618-622.

16. Friedland $B$, Metson R. A guide to recognizing maxillary sinus pathology and for deciding on further preoperative assessment prior to maxillary sinus augmentation. Int $\mathrm{J}$ Periodontics Restorative Dent. 2014 Nov-Dec;34(6):807-815. doi: 10.11607/prd.2043.

\section{Corresponding author:}

Tihomir Georgiev

18 Hristo Popovich Street

Varna 9000

Bulgaria

mobile phone: +359898614905

e-mail: tgeorgievphd@yahoo.com 